\title{
Assessment of the dysexecutive syndrome in schizophrenia
}

\author{
J. J. EVANS, ${ }^{1}$ S. E. CHUA, P. J. MCKENNA AND B. A. WILSON \\ From the MRC Applied Psychology Unit and Fulbourn Hospital, Cambridge; and the \\ Institute of Psychiatry, London
}

\begin{abstract}
Background. Cognitive neuropsychological theories hypothesize a role for frontal lobe executive deficits in the aetiology of schizophrenic symptoms. The study examined the performance of a schizophrenic group on the Behavioural Assessment of the Dysexecutive Syndrome (BADS; Wilson et al. 1996), a test battery which assesses the 'everyday' difficulties associated with the dysexecutive syndrome. Performance of the schizophrenics was contrasted with that of brain injured and healthy volunteer groups.

Methods. Matched groups of 31 schizophrenic patients, 35 patients with brain injuries and 26 healthy volunteers were administered the BADS. Patients were also given tests of general intelligence and memory. Patients and their relatives/carers also completed a questionnaire rating day-to-day failures of executive functioning.

Results. Schizophrenic and brain-injured patients showed impairment on the BADS, compared to healthy controls. There were no significant differences between the two patient groups. Significant impairment was found in a subgroup of 16 schizophrenics who showed otherwise intact general intellectual functioning, suggesting the existence of a specific executive deficit. Among the schizophrenic patient group there was evidence of a dissociation between executive and memory impairments. A significant correlation existed between performance on the BADS and relatives ratings of executive problems for the brain injured group, but not for the schizophrenic group.

Conclusions. The BADS is a useful tool for identifying executive deficits in people with a diagnosis of schizophrenia, especially those who are otherwise generally intellectually intact. This is particularly important in the context of rehabilitation and community transition programmes.
\end{abstract}

\section{INTRODUCTION}

In recent years schizophrenia has come to be regarded as a primarily biologically determined disorder. Consequently, genetic, biochemical and neuroanatomical/neurophysiological approaches to its aetiology have been joined by attempts to understand its symptoms in neuropsychological and cognitive neuropsychological terms. Empirical findings from post mortem (see Shapiro, 1993) and structural and functional imaging studies (see Chua \& McKenna, 1995), as well as theorizing from neurodevelopmental (e.g. Weinberger, 1987), neuroanatomical (e.g.

1 Address for correspondence: Mr J. J. Evans, The Oliver Zangwill Centre, Princess of Wales Hospital, Lynn Road, Ely, Cambs. CB6 1DN
Gray et al. 1991) and neurochemical (e.g. McKenna, 1987) standpoints, have repeatedly identified the frontal lobes as a likely site of abnormality. Correspondingly, a disorder of executive function has loomed large in accounts of the neurosychology of the disorder.

In the most thoroughly articulated cognitive neuropsychological account of schizophrenia, Frith (1992) has proposed that three principal cognitive abnormalities could underlie all its major signs and symptoms: a disorder of willed action, a disorder of self-monitoring and disorder of monitoring the intentions of others. $\mathrm{He}$ argues that a disorder of willed action, in particular, can account for symptoms which are common to both schizophrenic patients and patients with frontal lobe lesions, for example, 
lack of volition, perseveration and so-called utilization behaviour. These abnormalities in turn can be plausibly understood as a failure at the level of the Supervisory Attentional System (SAS) in the Norman \& Shallice (1986) model of the control of action, and they also form core features of the 'dysexecutive syndrome', the term proposed by Baddeley (1986) as a more functional characterization of the cognitive deficits traditionally associated with the frontal lobe syndrome.

The SAS is construed as being necessary for effective control of action in a number of situations: situations that involve planning or decision making; situation that involves error correction or troubleshooting; situations where responses are not well learned or contain novel sequences of actions; situations judged to be dangerous or technically difficult; and finally situations that require the overcoming of a strong habitual response or resisting temptation.

Evidence for a dysexecutive syndrome in schizophrenia has been sought in a number of neuropsychological studies. Schizophrenic patients have regularly been found to be impaired on tests such as the Wisconsin Card Sorting Test, verbal fluency, design fluency and other tasks sensitive to a frontal lesions (Kolb \& Whishaw, 1983; Goldberg et al. 1987, 1988; Liddle \& Morris, 1991; Allen et al. 1993; Beatty et al. 1993, 1994; Franke et al. 1993). It is typically assumed in these studies that such deficits are specific in the sense that they are present over and above any general intellectual impairment; in fact, however, none of these studies attempted to partial out the effects of general poor performance, and at least one (Beatty et al. 1993) found that impairment on the WCST was associated with comparable degrees of impairment on tests of memory and overall intellectual function.

The studies that have attempted to disentangle specific executive from generalized intellectual impairment in schizophrenia have offered conflicting findings: of four group studies, one (Crawford et al. 1993) found clear evidence for a disproportionate deficit in verbal fluency performance, two more (Braff et al. 1991; Morrison-Stewart et al. 1992) found that schizophrenic patients were impaired on some, but not all of a number of executive tasks, and one (Saykin et al. 1991) concluded that there was no evidence for a selective frontal impairment. In contrast, a study applying the neuropsychological case study approach to five chronically hospitalized patients provided a strong argument for the pre-eminence of frontal/executive deficits, which could be seen against a background of no, some or a great deal of general intellectual impairment (Shallice et al. 1991).

One reason for these mixed results may be that existing frontal tests may in fact be sufficiently sensitive to all aspects to all aspects of executive functioning. Patients with frontal lobe lesions have been described who display many of the characteristics of a dysexecutive syndrome in their daily lives, but who show no impairment on neuropsychological tests, including those considered sensitive to frontal lesions (Eslinger \& Damasio, 1985; Shallice \& Burgess, 1991). As a consequence, Shallice \& Burgess (1991) developed two tasks which they hypothesized would require functioning of a supervisory attentional system for successful completion. The tests were the Six Elements Test, which requires subjects to organize their activities in order to carry out six tasks in a limited time period and without breaking certain rules, and the Multiple Errands Test, which is carried out in a shopping centre and involves subjects having to buy certain objects, find out various pieces of information, be at a particular place at a certain time and adhere to various test rules given to them. Shallice \& Burgess (1991) were able to demonstrate that these tests were sensitive to the everyday 'dysexecutive' problems experience by three head-injured patients.

Taking the concept of 'everyday' executive impairment further Wilson et al. (1996) have incorporated a modified version of the Six Elements Test into a battery of tests, the Behavioural Assessment of the Dysexecutive Syndrome (BADS), designed to assess executive deficits in a way that reflects the difficulties such patients have in daily life. In an early pilot study (Alderman et al. 1993) and in a subsequent validation study described in the test manual (Wilson et al. 1996) it was demonstrated that the performance on the battery by brain-injured patients was predictive of the ratings given by relatives of day-to-day dysexecutive problems.

In this study we examined the performance of a group of schizophrenic patients on the BADS battery, and contrasted their performance with 
that of an age and IQ matched group of braininjured patients and an age and IQ matched healthy volunteer control group. One reason for the inclusion of the brain-injured group was simply to compare the performance of a group of people with a putative, but relatively unspecified brain disorder (the schizophrenic group) with a matched group of people with clearly documented brain damage. In addition, previous work (Wilson et al. 1996) has demonstrated that performance (by brain-injured patients) on this battery correlates with ratings of the extent of day-to-day executive functioning failures and therefore we wanted to compare the predictive power of performance on the BADS in two well matched groups of schizophrenic and brain-injured patients. We also examined the ability of the BADS to identify a specific executive impairment, that is one which is present over and above any co-existing general intellectual impairment, and one which is dissociable from another probable disproportionate neuropsychological deficit in schizophrenia, namely memory impairment (e.g. McKenna et al. 1995).

\section{METHOD}

\section{Subjects}

The subjects were 31 schizophrenic patients, 35 brain-injured patients and 26 healthy controls. All three groups were matched for age and estimated pre-morbid IQ (see Table 1). The 31 schizophrenic patients all met Research Diagnostic Criteria for schizophrenia (Spitzer et al. 1978); in addition, none was suffering from any co-existing illness that could have affected the brain and none had any history of head injury, alcohol or drug abuse. All the schizophrenic patients had chronic illnesses, but the overall severity ranged from mild-to-moderate (i.e. patients who were able to live independently in between hospital admissions), to relatively severe (i.e. patients who required intensive community support, lived in sheltered accommodation or were chronically hospitalized). None of the schizophrenic patients showed overall intellectual impairment to the extent that they scored below 24/30 on the Mini-Mental State Examination (MMSE) (Folstein et al. 1975), a cut-off which has been established as providing a clinically useful threshold for presence of mild dementia (Anthony et al. 1982). It should, however, be noted that many of the patients showed a considerable discrepancy between estimates of pre-morbid IQ and current IQ. All of the schizophrenic patients were taking neuroleptic medication at the time of testing.

The brain-injured patients were heterogeneous in terms of aetiology (including head injury, stroke, viral encephalitis and tumour), severity of injury (though all would fall into the moderate to severe range) and lesion location (though damage was more likely to be anterior-frontal/ temporal, than more posterior). As with the schizophrenic group levels of independence also varied with some patients being completely independent and others dependent upon carers. None had been diagnosed (pre- or post-injury) as suffering from serious psychiatric disorder.

\section{Procedure}

\section{Executive function}

The Behavioural Assessment of the Dysexecutive Syndrome (BADS) battery (Wilson et al. 1996) consists of six tests and a questionnaire. General details of the test are provided here, but more detailed descriptions of tests, administration and scoring methods can be obtained from the test manual. For each of the subtests a 'summary profile score' is obtained, (with a maximum of

Table 1. Subjects

\begin{tabular}{|c|c|c|c|}
\hline & $\begin{array}{l}\text { Schizophrenic } \\
\text { patients }\end{array}$ & $\begin{array}{l}\text { Brain-injured } \\
\text { patients }\end{array}$ & Healthy controls \\
\hline$N$ & 31 & 35 & 26 \\
\hline Mean age (years) & $38 \cdot 9$ (S.D. $9 \cdot 85$ ) & $40 \cdot 4$ (S.D. $15 \cdot 49$ ) & $39 \cdot 1$ (s.D. $19 \cdot 02$ ) \\
\hline $\begin{array}{l}\text { Mean estimated pre- } \\
\text { morbid (NART) IQ }\end{array}$ & $\begin{array}{r}109 \cdot 35 \text { (S.D. 9.82) } \\
\text { (Range 91-124) }\end{array}$ & $105 \cdot 0$ (S.D. $12 \cdot 58)$ & $110 \cdot 3$ (s.D. $8 \cdot 95$ ) \\
\hline $\begin{array}{l}\text { Mean-Mini-Mental } \\
\text { Score }\end{array}$ & $\begin{array}{l}27 \cdot 93 / 30 \\
\text { (Range 25-30) }\end{array}$ & - & - \\
\hline Mean current IQ & $\begin{array}{l}95 \cdot 0 \text { (S.D. 14.00) } \\
\text { (Range 69-122) }\end{array}$ & - & - \\
\hline
\end{tabular}


four and minimum of zero) and these are summed to produce an overall battery profile score (out of 24).

\section{Rule Shift Cards}

In this test, which examines the ability of subjects to shift from the use of one simple rule for responding to another more complex rule, the subject has to respond 'Yes' or 'No' when a series of 20 playing cards are turned over one at a time. In the first version of the task the subject has to use the rule, 'say yes to red and no to black', and in the second version they must use the rule, 'say yes if the card is the same colour as the last one, otherwise say no'. Time taken and the number of errors made are recorded.

\section{Action Program}

This is a novel problem solving task based on a description of a task by Klosowska (1976). The subject is presented with a set of materials; a large round container, half full of water, with a removable lid that has a small hole in the top; a tall thin container with a cork loose in the bottom of the tube; a piece of shaped stiff wire which is not long enough to reach the cork; and a small cylindrical tube to which a top can be attached. Subjects are required to remove the cork from the tall tube, making use of the props provided and without touching the main assembly with their hands (the wire is used to remove the lid from the large container, the top is attached to the small cylindrical tube and this is used take water from the large container and pour it into the tall tube in order to float the cork to the top), A score based on the number of steps completed without prompting is recorded.

\section{Key search}

The development of this test was influenced by a test from the Stanford Binet battery. The subject is provided with a piece of paper with a large square drawn on it. They are asked to imagine the square is a large field and they must draw the path they would take to search this field in order to find some lost keys. A score is awarded according to the functionality of the search pattern adopted.

\section{Temporal judgement}

In this test subjects are asked four questions which require them to estimate the time length of some activity. It is emphasized to subjects that there is no absolutely correct answer and that the task is concerned with subjects ability to produce a 'sensible guess'. The questions are 'How long does a routine dental appointment take?', 'How long does it take a window cleaner to clean the windows of an average size house?', 'How long do most dogs live for?' and 'How long does it take to blow up a party balloon?'. Answers are scored according to the degree of deviation from the answers most commonly provided by the BADS battery normative sample of 216 controls.

\section{Zoo map}

Subjects are given a map of zoo and a set of instructions relating to places they have to visit and rules they must follow (such as not using some paths twice). In the first part of the test, the order in which the visits should take place is not specified and thus the subject is required to plan a route which enables them to visit all the places without breaking any rules. In the second part another identical map is provided, but the order in which places should be visited is specified. Thus, the main difference between the tasks is the considerably greater planning element of the first task. Scoring is based on the number of errors made, the number of places visited in the correct sequence and also the time taken to plan and execute the plan.

\section{Modified Six Elements}

This task is a simplified version of the original Shallice \& Burgess (1991) task and involves the subject being given instructions to do three tasks (dictation, simple arithmetic and picture naming), each of which has two sections, A and B. The subject has $10 \mathrm{~min}$ in which to do at least some of all six sections, but they are not allowed to do so sections A and B of the same task one after the other. Once it is clear that the subject has understood the test instructions, they begin the test and their performance is monitored. Scores used for analysis are the number of tasks attempted, the number rule breaks made and the maximum time spent on any one task.

In addition, each of the subjects and a carer or relative were administered the Dysexecutive (DEX) Questionnaire. This contains 20 items and is constructed so as to sample the range of cognitive, behavioural and emotional problems commonly associated with the dysexecutive 
syndrome. Items include statements such as 'I act without thinking, doing the first thing that comes to mind', 'I have difficulty thinking ahead and planning for the future', and 'I find it difficult to keep my mind on something and am easily distracted.' Each item is scored on a 5 point (0-4) Likert scale (ranging from 'Never' to 'Very Often'). One version is designed to be completed by the patient and another by a relative or carer. An overall impairment score is derived from totalling the 20 individual item scores (the maximum score is 80 ).

\section{Memory}

The Rivermead Behavioural Memory Test (RBMT) (Wilson et al. 1985) was also administered to the schizophrenic and braininjured patients. Like the BADS, this is a test designed to be sensitive to 'everyday' memory impairment, in particular long-term/secondary memory. It consists of 12 subtests, covering recall and recognition, remembering a route, orientation and three measures of prospective memory, the ability to remember to do things; each of these is passed or failed to give a screening score of up to 12 .

Normative data on the RBMT have been obtained on 118 normal individuals, and the test has also been validated against 176 patients with brain injury. Screening score ranges for normal, poor, moderately and severely impaired memory have been established.

\section{RESULTS}

\section{Executive function in the three groups}

Table 2 shows the mean summary profile scores for the individual subtests, the overall BADS profile score and scores on the DEX questionnaire for all three groups. Fig. 1 illustrates the scores of the individual subtests. Error bars indicate one standard deviation from the mean, and illustrate the considerable variability in performance, especially among the two patient groups. Fig. 2 shows the percentage of each of the three main study groups (plus a subsample of schizophrenics who show relatively little general intellectual decline from estimates of pre-morbid IQ, discussed in the following section) falling into the various BADS performance categories (ranging from Impaired through to Superior). The performance by each group on each of the BADS tasks, as well as the overall BADS profile score and DEX questionnaire ratings, was compared using ANOVA and planned post hoc Scheffé tests.

The overall BADS profile score ANOVA revealed highly significant differences between the groups, with Scheffé's tests revealing significant differences between schizophrenics and controls as well as brain-injured patients and controls, but no difference between the schizophrenic and brain-injured groups. As shown in Table 2 the post hoc tests revealed significant differences between the schizophrenic group and the control group on many, though not all of the tasks in the BADS battery. There were also differences between the brain-injured group and the controls on the same set of tasks. There were, however, no differences between the schizophrenic and the brain-injured groups on any of the BADS tasks. In fact, the only differences between the two patient groups was on the scores for the self-rated DEX questionnaire, where the schizophrenic patients gave significantly lower ratings of their own dysexecutive impairment than the brain-injured patients.

\section{Are the BADS deficits in schizophrenic independent of general intellectual impairment?}

Although the schizophrenic patients were matched for estimated pre-morbid IQ with the normal controls, and none of them could be described as generally demented, a number did show a significant decrease in their current IQ in comparison to the NART estimated IQ. The question, therefore, arises as to whether the poor BADS performance found in the schizophrenic group was simply the consequence of a more general impairment affecting many or all cognitive functions.

We addressed this issue by examining the performance of a subsample of the schizophrenic patients who showed relatively little IQ deterioration. Sixteen of the schizophrenic patients had a discrepancy in NART estimated IQ and WAIS IQ of less than 15 points (mean age 40.0 years, mean NART IQ 108.6, mean current IQ 103.3). This group was matched with 16 of the healthy controls (mean age 37.0 years, mean NART IQ 105.1). There was no significant difference between the NART IQs for the two groups $(t=1 \cdot 24, P=0.225)$, and in addition the 
Table 2. Mean scores (and standard deviations) and ANOVA results for schizophrenic, braininjured and healthy control groups on the BADS subtests, overall BADS profile score and the DEX Questionnaire

\begin{tabular}{|c|c|c|c|c|c|}
\hline \multirow[b]{2}{*}{ Test } & \multirow{2}{*}{$\begin{array}{l}\text { Schizophrenic } \\
\quad(N=31)\end{array}$} & \multirow{2}{*}{$\begin{array}{l}\text { Brain injured } \\
\quad(N=35)\end{array}$} & \multirow{2}{*}{$\begin{array}{l}\text { Healthy } \\
\text { controls } \\
(N=26)\end{array}$} & \multicolumn{2}{|c|}{ ANOVA } \\
\hline & & & & $\mathrm{F}$ & $\mathrm{P}$ \\
\hline Rule shift cards & $2 \cdot 94(1 \cdot 29)$ & $2 \cdot 85(1 \cdot 42)$ & $3.62(0.75)$ & $3 \cdot 335$ & $0 \cdot 040$ \\
\hline Action program & $2.73(1.34)^{*}$ & $3 \cdot 12(1 \cdot 25)^{*}$ & $3.89(0.33)$ & $7 \cdot 768$ & 0.001 \\
\hline Key search & $1.83(1.23)^{*}$ & $2.03(1.49)^{*}$ & $3.00(1 \cdot 33)$ & $5 \cdot 789$ & $0 \cdot 004$ \\
\hline Temporal judgement & $1.84(1.13)^{*}$ & $1.69(0.93)^{*}$ & $2 \cdot 5(0 \cdot 65)$ & $5 \cdot 910$ & 0.004 \\
\hline Zoo map & $1.00(1.41)$ & $1.20(1.41)$ & $1.72(1.49)$ & $2 \cdot 002$ & $0 \cdot 141$ \\
\hline Modified six elements & $2 \cdot 27(1 \cdot 20)^{*}$ & $1.65(1.28)^{*}$ & $3.52(0.79)$ & 18.657 & $<0.0001$ \\
\hline BADS profile score & $12.79(4.58)^{*}$ & $12 \cdot 44(5.08)^{*}$ & $18.00(2.95)$ & $12 \cdot 51$ & $<0.0001$ \\
\hline DEX carer's rating & $31.52(15.03)^{*}$ & $33.97(15 \cdot 85)^{*}$ & $11 \cdot 25(6 \cdot 71)$ & $15 \cdot 23$ & $<0.0001$ \\
\hline DEX self-rating & $18.07(10.93) \dagger$ & $27 \cdot 59(14 \cdot 77)$ & $21 \cdot 81(8 \cdot 16)$ & $4 \cdot 25$ & $0 \cdot 017$ \\
\hline
\end{tabular}

* Significantly different from controls using post-hoc Scheffé tests $(P<0 \cdot 05)$.

$\dagger$ Significantly different from brain injured using post-hoc Scheffé test $(P<0 \cdot 05)$.

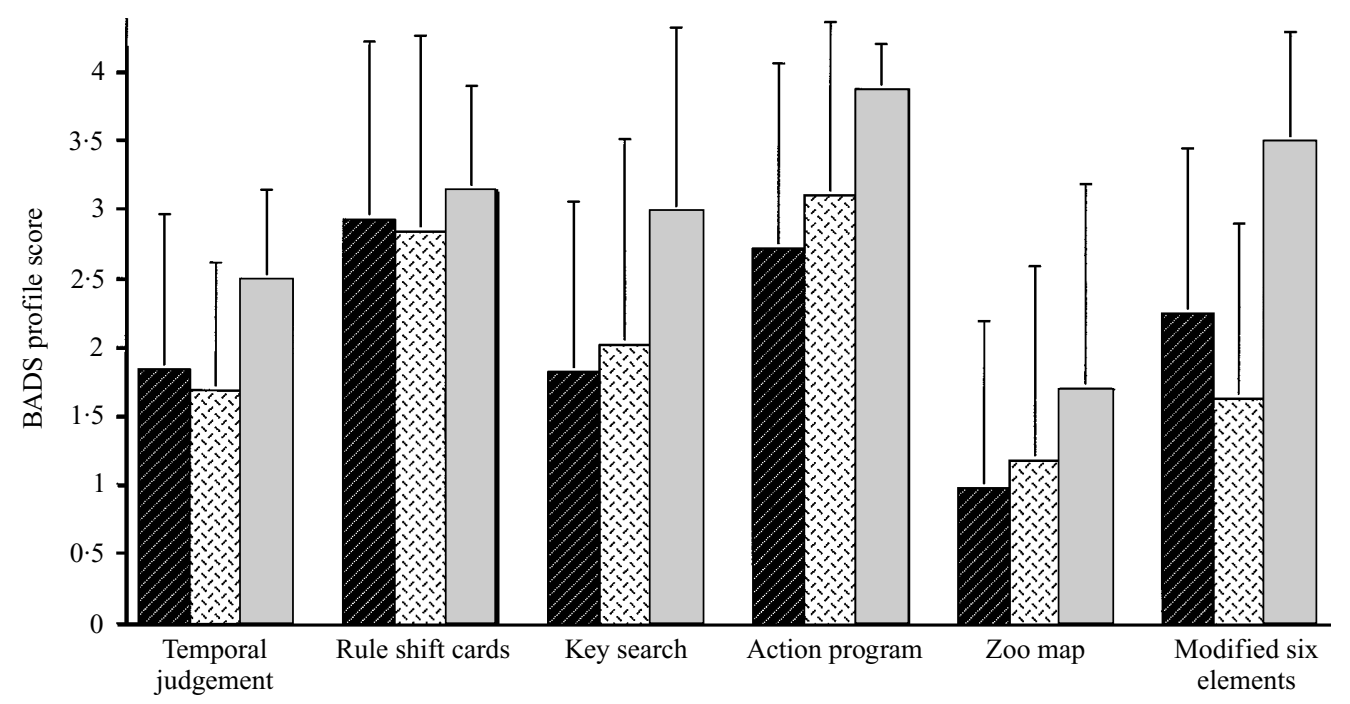

FIG. 1. Individual BADS subtest profile scores for schizophrenic (因), brain-injured (团) and healthy control ( $\square$ ) groups.

current IQ of the schizophrenics was not significantly different to the NART estimated IQ for the controls $(t=-0.58, P=0.566)$.

Fig. 2 shows the percentage of the subsample of schizophrenics with IQ decline of less than 15 points falling into each of the BADS performance categories. The performance of the 16 schizophrenics and 16 matched healthy controls on the six BADS tests was compared using individual $t$ tests (ANOVA and conservative post-hoc tests were felt to be unnecessary as this analysis was confirmatory). As shown in Table 3 , significant differences continued to be evident on three of the tasks (Temporal Judgement, Action Program and Modified Six Elements).
The Rule Shift Cards test and the Key Search were not significantly different between these two groups (but had been for the whole sample), and the Zoo Map continued to show no significant difference. The overall BADS profile score remained significantly different between the two groups.

\section{Is the executive impairment independent of memory impairment?}

As noted, a consistent finding in neuropsychological studies of schizophrenia is that of significant memory impairment in many patients. In addition to the intrinsic interest in the relationship between executive and memory 


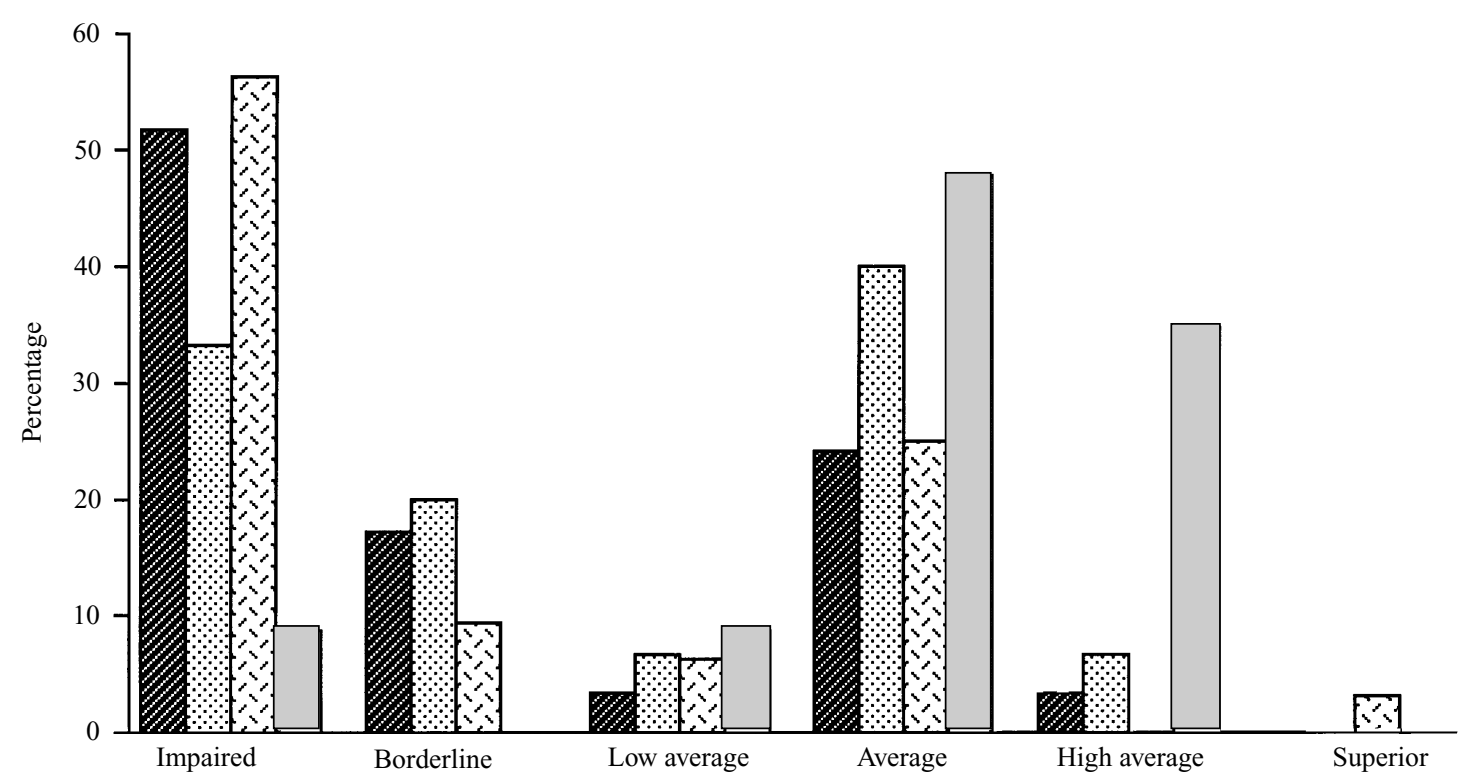

FIG. 2. Percentage of subjects(schizophrenics(wholegroup)(国); schizophrenicsubsample(intellect preserved)(圈); brain-injured(団); healthy controls $(\square)$ in each of the BADS performance categories.

Table 3. Mean summary profile cores (and standard deviations) and t-test results on the BADS subtests, overall BADS profile score and the DEX Questionnaire for a subsample of intellectually preserved schizophrenic patients $(\mathrm{N}=16)$ and matched healthy controls $(\mathrm{N}=16)$

\begin{tabular}{|c|c|c|c|c|}
\hline Test & $\begin{array}{l}\text { Schizophrenics } \\
\quad(N=16)\end{array}$ & $\begin{array}{l}\text { Healthy controls } \\
\quad(N=16)\end{array}$ & $t$ & $P$ \\
\hline Rule shift cards & $3.44(1.21)$ & $3.5(0.89)$ & $-0 \cdot 166$ & $0 \cdot 869$ \\
\hline Action program & $3.00(1.21)$ & $3.88(0.34)$ & -2.782 & 0.009 \\
\hline Key search & $2 \cdot 06(1 \cdot 18)$ & $2 \cdot 88(1.36)$ & -1.804 & 0.081 \\
\hline Temporal judgement & $1.88(1.03)$ & $2.63(0.62)$ & $-2 \cdot 51$ & 0.018 \\
\hline Zoo map & $1.25(1.24)$ & $1.25(1.07)$ & 0 & - \\
\hline Modified six elements & $2.33(1.23)$ & $3 \cdot 27(0 \cdot 88)$ & $-2 \cdot 381$ & 0.024 \\
\hline BADS profile score & $14.47(3.29)$ & $17.33(2.58)$ & -2.654 & 0.013 \\
\hline
\end{tabular}

deficits in schizophrenia, we were also interested in whether the memory impairment might be confounding the results on the executive tasks, as has recently been claimed for the Wisconsin Card Sorting Test (Corcoran \& Upton, 1993). In particular, we were concerned to establish that performance on the Modified Six Elements task was not being confounded by memory impairment - in this test, although the subject always has access to the instructions and a number of pure amnesic patients have performed satisfactorily on the task, it could be argued that the demands of remembering the overall task goals could interfere with performance.

We, therefore, examined data from the subgroup of intellectually preserved schizophrenic patients to establish whether there was any correlation between performance on the RBMT and the Modified Six Elements Test. Performance on this task might intuitively be expected to be sensitive to memory impairment and thus would represent a conservative test of the potential confounding effect of a memory impairment on a test of executive functioning. The mean RBMT screening score for the schizophrenic group was 6.57 (S.D. 1.91) with a range from 3-10 points, with $50 \%$ of the group falling into the 'moderately impaired' range, $42.8 \%$ in the 'poor memory' range and only $7 \cdot 2 \%$ falling in the normal memory range. For the Modified Six Elements test we used the summary profile score. The correlation between RBMT screening 
Table 4. Heterogeneity of performance of schizophrenic patients on the Rivermead Behavioural Memory Test and the Modified Six Elements Test

\begin{tabular}{llrrrr}
\hline \hline Subject & \multicolumn{2}{c}{ R.C. } & H.C. & D.F. & T.G. \\
\hline Age (years) & 36 & 38 & 30 & 37 \\
NART IQ & 117 & 124 & 110 & 107 \\
Current IQ & 115 & 112 & 109 & 96 \\
RBMT* & Impaired & Impaired & Unimpaired & Unimpaired \\
Six Elements† & Unimpaired & Impaired & Unimpaired & Impaired \\
\hline \hline
\end{tabular}

* Impaired performance defined by screening score $\leqslant 6$.

$\dagger$ Impaired performance defined by summary profile score $<2$.

score and the Modified Six Elements profile score was $-0.09(P=0.79)$, suggesting that memory impairment could not account for poor performance on the test of executive functioning.

RBMT data (standardized profile scores) for the brain injured group were also available, with scores ranging from 1 (severely impaired) to 24 (normal). The correlation between RBMT standardized profile scores and Modified Six Elements profile score was $0.256(P=0 \cdot 20)$ for this group, providing additional evidence that poor performance on the Modified Six Elements test cannot be attributed to poor memory functioning.

Another way of exploring this issue is by single case dissociation methodology (Shallice, 1988; Shallice et al. 1991), which argues that if one patient is impaired in relation to a control group on one task, but normal on a second task, and in contrast a second patient shows the reverse pattern of impairment, then those two tasks must be relying on different processing resources for successful completion. Among our schizophrenic sample it was possible to identify four patients who demonstrated the complete range of possible combinations of performance on the Modified Six Elements task and the Rivermead Behavioural Memory Task. Table 4 illustrates this diversity of performance, with the double dissociation in task performance being demonstrated by patients R.C. and T.G., with the other results combinations (i.e. unimpaired or impaired on both tasks) being shown by patients H.C. and D.F.

\section{The relationship between performance on the BADS and ratings of executive impairment}

As shown in Table 2, neither the schizophrenic nor the brain injured patients showed significantly different self-ratings from the controls on the DEX questionnaire. In contrast, both patient groups showed significant impairment compared to controls on the carer's version of the DEX questionnaire. We were interested to know whether there would be a relationship between scores on the BADS battery of tests and the ratings of 'everyday executive impairment' provided by the patients themselves and also by their relatives/carers on the DEX questionnaire.

There were no significant correlations whatsoever between any of the BADS subtest scores, or the overall BADS profile score and selfratings on the DEX questionnaire for the schizophrenic group or the brain injured group. Correlations between the scores on the individual BADS tasks as well as the overall BADS profile score and relatives'/carers' ratings on the DEX for the schizophrenic patients and the brain injured group are shown in Table 5 (data for the whole sample and the subgroup of 16 patients are provided separately). Results for the brain-injured group demonstrate significant correlations between three of the subtests (and trends to significance for an additional two other subtests) and the DEX questionnaire ratings. There is also a strong correlation between the overall BADS profile score and the DEX ratings. In contrast, for the schizophrenic groups, with the exception of the Zoo Map test, there is no clear relationship between BADS test performance and DEX ratings. One measure from the Modified Six Element Test used in the calculation of the summary profile score, the number of subtasks attempted, did correlate with relatives' DEX ratings, the correlations being $-0.443(P=0.020)$ for the whole schizophrenic sample and $-0.612(P=0.018)$ for the subsample of 16 intellectually intact patients. However the other main measure used to calculate the profile score, the number of rule- 
Table 5. Correlation between BADS tests and DEX Questionnaire (relatives ratings) scores

\begin{tabular}{|c|c|c|c|}
\hline \multirow[b]{2}{*}{ Test } & $\begin{array}{c}\text { Schizophrenics } \\
\text { (whole group) } \\
(N=31)\end{array}$ & $\begin{array}{l}\text { Schizophrenic } \\
\text { subgroup } \\
(N=16)\end{array}$ & $\begin{array}{l}\text { Brain-injured } \\
\quad(N=35)\end{array}$ \\
\hline & $P$ & $P$ & $P$ \\
\hline Rule shift cards & $-0.236(0.228)$ & $-0.173(0.546)$ & $-0.374(0.037)^{*}$ \\
\hline Action program & $-0 \cdot 104(0 \cdot 601)$ & $-0 \cdot 371(0 \cdot 177)$ & $-0.326(0.068)$ \\
\hline Key search & $0 \cdot 124(0.533)$ & $0.215(0.449)$ & $-0.302(0.099)$ \\
\hline Temporal judgement & $-0.081(0.683)$ & $0 \cdot 241(0 \cdot 394)$ & $-0 \cdot 270(0 \cdot 143)$ \\
\hline Zoo map & $-0.407(0.031)^{*}$ & $-0.616(0.013)^{*}$ & $-0.388(0.025)^{*}$ \\
\hline Modified six elements & $-0.298(0 \cdot 133)$ & $-0 \cdot 449(0 \cdot 109)$ & $-0.430(0.013)^{*}$ \\
\hline BADS profile score & $-0.280(0 \cdot 159)$ & $-0.389(0 \cdot 174)$ & $-0.566(0.001)^{*}$ \\
\hline
\end{tabular}

* $P<0.05$.

breaks made did not correlate at all with the relatives ratings $(r=-0.050, P=0.807$ for whole sample and $r=-0.319, P=0.272$ for subsample of 16). In contrast the rule breaks measure did correlate with DEX rating for the brain-injured group $(r=0.355, P=0.0498)$.

For the whole schizophrenic sample there was no significant correlation between the NART estimated pre-morbid IQ and DEX ratings, but the measure of current IQ (WAIS) did correlate significantly with the relatives' DEX ratings $(r=0.438, P=0.021)$. for the subsample of 16 schizophrenics neither the pre-morbid IQ nor the current IQ measures correlated significantly with DEX ratings.

\section{DISCUSSION}

The results of this study indicate that the performance of relatively chronically and severely ill schizophrenic patients on a series of tasks sensitive to 'everyday' executive impairment is similar to that of patients with moderateto-severe brain injury. More detailed analysis suggests that the impairment is not a consequence of an across-the-board decline in intellectual functioning, but rather appears, at least in some cases, to involve a specific impairment in planning and problem solving.

In a study providing what is perhaps the strongest evidence for a specific impairment of executive function in schizophrenia, Shallice et al. (1991) argued that their detailed assessment of five schizophrenic patients pointed to the conclusion that, "all chronic schizophrenics have problems with processes tapped by "frontal" tests and that some schizophrenics have, in addition, a more widespread cognitive impairment'. Data from the present study certainly support a conclusion that many patients diagnosed as schizophrenic have problems with tasks involving planning, monitoring, switching tasks and problem solving in novel or unusual situations. Nevertheless, as in other group studies of schizophrenia, there was very considerable heterogeneity in performance. Even on the Six Elements Test, which has been found to be an exceptionally sensitive test of the integrity of the skills traditionally thought of as 'executive' functions, some of the schizophrenic patients (approximately $35 \%$ ) managed to carry out the necessary strategic planning and switching very successfully (having scores within one standard deviation of the control subjects performance). This certainly cast doubts on the hypothesis that all schizophrenics are impaired on executive tests, although of course it has to be acknowledged that neither the Six Elements task, nor any of the other test included in the BADS battery, can be said to test all the hypothesized functions of the Supervisory Attentional System. Perhaps the decisive test of Shallice et al.'s (1991) proposal that all chronic schizophrenic patients will show some degree of executive impairment would be to examine the performance of chronic schizophrenic patients on the complex scheduling task, the Multiple Errands Test.

The lack of correlation between BADS test and memory test scores, and also the single case data, provide evidence that executive impairment is dissociable from memory impairment in schizophrenia. This finding is also in contrast to that of Shallice et al. (1991): they found relatively 
little evidence of memory impairment in the absence of overall intellectual impairment in their five chronic schizophrenic patients, and argued that the patchy poor memory performance that was present could be understood as the consequence of a co-existing executive deficit. The present study, together with another group study (Elliott et al. 1995) and a further single case study (Laws et al. 1997), suggest that this is no longer a tenable position. Schizophrenia appears to be characterized by at least two separate areas of specific (i.e. disproportionate to the overall level of intellectual impairment); executive function and memory.

In this study, we did not examine the relationship between the executive impairment and schizophrenic symptomatology. Despite observations of phenomena resembling negative symptoms and formal thought disorder in patients with frontal lobe lesions (Liddle, 1987), and many theoretical proposals arguing for such an association (e.g. Gray et al. 1991; McGrath, 1991; Frith, 1992), the empirical findings in this area are far from consistent. Liddle (1987; Liddle \& Morris, 1991) found correlations between both negative symptoms and disorganization symptoms (i.e. formal thought disorder) and poor performance on various executive tests, but also poor performance on non-executive tests. Morrison-Stewart et al. (1992) found no relationship between negative symptoms and executive function, and two further studies (Mortimer et al. 1997; Clark \& O'Carroll, 1997) found no correlations between performance on executive tests and schizophrenic symptomatology. Once again, however, this might be a consequence of the limitations of current formulations of frontal lobe executive functioning and consequentially a lack of tools to specifically assess such functions. For example, Frith \& Done (1989) and Mlakar et al. (1994) have found evidence of a relationship between a highly specific form of executive failure, impaired self-monitoring of actions, and first-rank symptoms involving experiences of alienation of one's own thoughts and actions.

If we assume that the BADS battery is a good measure of executive functioning and if we also assume that the DEX questionnaire is a good measure of the degree of problems with executive functioning in day to day life then we would expect there to be a correlation between the two measures. Furthermore, since the performance of the schizophrenic and brain-injured groups was similar on both the BADS and the DEX, then we might expect the same relationship to exist between these measures for both of the groups. As has been previously found for a brain injured group (Alderman et al. 1993; Wilson et al. 1996), there was no relationship between performance on any of the BADS tasks and schizophrenics' self-ratings of their degree of everyday executive impairment. Since constructs such as 'self-monitoring' and 'insight' are central to the broad concept of executive functioning this is perhaps not surprising. By contrast, there was a significant relationship between the DEX ratings of executive impairment given by relatives of the brain injured patients and a number of the BADS tests as well as the overall profile score. However, for the schizophrenic group there was no such relationship with the overall profile score and only one test (Zoo Map) and one measure from another test (Number of tasks attempted on the Modified Six Elements Test) did correlate significantly with relatives/carers DEX ratings. Clark \& O'Carroll (1997) also found no relationship between executive function (performance on the Modified Six Elements) and REHAB (Baker \& Hall, 1984) status. One possible explanation for these findings is that while many people diagnosed as schizophrenic might have executive impairments (to which the BADS battery of tests is sensitive and which do indeed cause the sort of everyday problems identified by the DEX questionnaire), certain schizophrenic symptoms might not be related to any executive impairment (or alternatively are caused by some form of executive impairment to which the BADS is not sensitive), but such symptoms may themselves cause some of the day to day problems sampled by the DEX questionnaire. Another possibility is that the mechanisms giving rise to poor performance on executive tasks are different in schizophrenia to those in brain injury; in other words, while some process in schizophrenia compromises neuropsychological performance, this process may be sufficiently different from deficits caused by the gross neurological destruction associated with brain injury to prevent the deficit translating into everyday impairments in the same way. It is clear that this issue requires further exploration. Certainly schizo- 
phrenic patients can be found who fail executive tasks, but do not show many of the features typically associated with a frontal lobe dysexecutive syndrome, in the same way that many chronic schizophrenic patients show moderate or marked impairment on memory tests, but memory deficits are rarely commented upon by relatives or carers.

From a more clinical perspective, especially from the perspective of psychiatric rehabilitation, it might be argued that identifying the specific nature of the underlying cognitive impairment causing an 'executive' impairment is less important than identifying the particular tasks with which the patient has difficulties. This study has demonstrated that a significant proportion of schizophrenic patients, including those who appear to be generally intellectually intact have problems with a range of more 'open-ended' planning and problem solving tasks. The heterogeneity of performance among the schizophrenic group is also important; a simple diagnosis of schizophrenia (of whatever subtype) is clearly not sufficient at this stage to make firm predictions about the neuropsychological strengths and weaknesses of the particular patient. Detailed assessment is always going to be necessary. This is especially important in the context of the 'community care' policies of many countries and in particular the emphasis on moving people with schizophrenia out of large institutions and into more independent living situations. Findings such as those from this study and others should alert clinicians to the possibility that, while the executive problems of some patients might be very obvious, for others the problem may be more subtle and even masked by the apparently successful way that such patients cope on a day to day basis in an institutional setting. Most institutional settings are highly routinized and may make few demands upon patients in terms of planning, organizing, and problems solving. Independent living situations may be dramatically different in these aspects and it may be that the presence of such impairments only becomes clear when patients are put into the new situation. Psychological stress is a well documented precipitant of relapse and the inability to deal with novel, non-routine situations is likely to be extremely stressful. This is not an argument against community care; it is an argument in favour of appropriate support and rehabilitation efforts. Careful assessment prior to movement is, therefore, called for and where possible rehabilitation work should be undertaken to reduce the demands placed upon an impaired supervisory attentional system. We hope that the BADS will be one tool that may help in the process of identifying such difficulties.

\section{REFERENCES}

Alderman, N., Evans, J. J., Burgess, P. \& Wilson, B. A. (1993). Behavioural assessment of the dysexecutive syndrome. Journal of Clinical and Experimental Neuropsychology 15, 69-70.

Allen, H. A., Liddle, P. F. \& Frith, S. D. (1993). Negative features, retrieval processes and verbal fluency in schizophrenia. British Journal of Psychiatry 163, 769-775.

Anthony, J. C., LeReche, L., Niaz, U., Von Korff, M. R. \& Folstein, M. F. (1982). Limits of the 'Mini-Mental State' as a screening test for dementia and delirium among hospital patients. Psychological Medicine 12, 397-408.

Baddeley, A. D. (1986). Working Memory. Oxford University Press: Oxford.

Baker, R. D. \& Hall, J. J. (1984). REHAB: Rehabilitation Evaluation Hall and Baker. Vine Publishing: Aberdeen.

Beatty, W. W., Jocic, Z., Monson, N. \& Katzung, V. M. (1994). Problem solving by schizophrenic and schizoaffective patients on the Wisconsin and California card sorting tests. Neuropsychology 8, 49-54.

Beatty, W. W., Jocic, Z., Monson, N. \& Staton, D. (1993). Memory and frontal lobe dysfunction in schizophrenia and schizoaffective disorder. Journal of Nervous and Mental Disease 181, 448-453.

Braff, D. L., Heaton, R., Kuck, J., Cullum, M., Moranville, J., Grant, I. \& Zisook, S. (1991). The generalised pattern of neuropsychological deficits in outpatients with chronic schizophrenia with heterogeneous Wisconsin Card Sorting Test results. Archives of General Psychiatry 48, 891-898.

Chua, S. E. \& McKenna, P. J. (1995). Schizophrenia - a brain disease? A critical review of structural and functional brain abnormality in the disorder. British Journal of Psychiatry 166, 563-582.

Clark, O. \& O'Carroll, R. E. (1997). An examination of the relationship between executive function and rehabilitation status in schizophrenia. Neuropsychological Rehabilitation (in the press).

Corcoran, R. \& Upton, D. (1993). A role for the hippocampus in card sorting? Cortex 29, 293-304.

Crawford, J. R., Obonswain, M. C. \& Bremner, M. (1993). Frontal lobe impairment in schizophrenia: relationship to intellectual functioning. Psychological Medicine 23, 787-790.

Elliott, R., McKenna, P. J., Robbins, T. W. \& Sahakian, B. J. (1995). Neuropsychological evidence for fronto-striatal dysfunction in schizophrenia. Psychological Medicine 25, 619-630.

Eslinger, P. J. \& Damasio, A. R. (1985). Severe disturbance of higher cognition after bilateral frontal ablation: patient EVR. Neurology 35, 1731-1741.

Folstein, M. F., Folstein, S. E. \& McHugh, P. R. (1975). MiniMental State:' a practical method for grading the cognitive state of patients for the clinician. Journal of Psychiatric Research 12, 189-198.

Franke, P., Maier, W., Hardt, J., Frieboes, R., Lichtermann, D. \& Hain, C. (1993). Assessment of frontal lobe functioning in schizophrenia and unipolar major depression. Psychopathology 26, 76-84.

Frith, C. D. (1992). The Cognitive Neuropsychology of Schizophrenia. Lawrence Erlbaum Associates: Hove. 
Frith, C. D. \& Done, D. J. (1989). Experiences of alien control in schizophrenia reflect a disorder in the central monitoring of action. Psychological Medicine 19, 359-363.

Goldberg, T. E., Weinberger, D. R., Berman, K. F., Pliskin, N. H. \& Podd, M. H. (1987). Further evidence for dementia of prefrontal type in schizophrenia: a controlled study of teaching the Wisconsin Card Sorting Test. Archives of General Psychiatry 44, 1008-1014.

Goldberg, T. E., Kelsoe, J. R., Weinberger, D. R., Pliskin, N. H., Kirwin, P. D. \& Berman, K. F. (1988). Performance of schizophrenic patients on putative neuropsychological tests of frontal lobe function. International Journal of Neuroscience 42, 51-58.

Gray, J. A., Rawlins, J. N. P., Hemsley, D. R. \& Smith, A. D. (1991). The neuropsychology of schizophrenia. Behavioral and Brain Sciences 14, 1-84.

Klosowska, D. (1976). Relation between ability to program actions and location of brain damage. Polish Psychological Bulletin 7, 245-255.

Kolb, B. \& Whishaw, I. Q. (1983). Performance of schizophrenic patients on tests sensitive to left or right frontal, temporal or parietal function in neurological patients. Journal of Nervous and Mental Disease 171, 435-443.

Laws, K., McKenna, P. J. \& McCarthy, R. A. (1997). Reconsidering the gospel according to group studies: a neuropsychological case study approach to schizophrenia. Cognitive Neuropsychiatry (in the press).

Liddle, P. F. (1987). Schizophrenic syndromes, cognitive performance and neurological dysfunction. Psychological Medicine 17, 49-57.

Liddle, P. F. \& Morris, D. L. (1991). Schizophrenic symptoms and frontal lobe performance. British Journal of Psychiatry 158, $340-345$.

McGrath, J. (1991). Ordering thoughts on thought disorder. British Journal of Psychiatry 158, 307-316.

McKenna, P. J. (1987). Pathology, phenomenology and the dopamine hypothesis of schizophrenia. British Journal of Psychiatry 151, 388-301.

McKenna, P. J., Clare, L. \& Baddeley, A. D. (1995). Schizophrenia. In Handbook of Memory Disorders (ed. A. D. Baddeley, B. A. Wilson and F. N. Watts), pp. 271-292. Wiley: Chichester.

Mlakar, J., Jensterle, J. \& Frith, C. D. (1994). Central monitoring deficiency and schizophrenic symptoms. Psychological Medicine 24, 557-564.
Morrison-Stewart, S. L., Williamson, P. C., Corning, W. C., Kutcher, S. P., Snow, W. G. \& Merskey, H. (1992). Frontal and non-frontal lobe neuropsychological test performance and clinical symptomatology in schizophrenia. Psychological Medicine 22, 353-359. Mortimer, A. M., Bentham, P. E., McKay, A. P., Quemada, I. \& McKenna, P. J. (1997). Delusions: a phenomenological and psychological exploration. Cognitive Neuropsychiatry (in the press).

Norman, D. A. \& Shallice, T. (1986). Attention to action: willed and automatic control of behaviour. In Consciousness and Selfregulation, Vol. 4 (ed. R. J. Davidson, G. E. Schwartz and D. Shapiro), pp. 1-18. Plenum Press: New York.

Saykin, A. J., Gur, R. C., Gur, R. E., Mozley, P. D., Mozley, L. H., Resnick, S. M., Kester, B. \& Stafiniak, P. (1991). Neuropsychological function in schizophrenia: selective impairment in learning and memory. Archives of General Psychiatry 48, 618-624.

Shallice, T. (1988). From Neuropsychology to Mental Structure. Cambridge University Press: Cambridge

Shallice, T. \& Burgess, P. (1991). Deficits in strategy application following frontal lobe damage in man. Brain 114, 727-741.

Shallice, T., Burgess, P. W. \& Frith, C. D. (1991). Can the neuropsychological case-study approach be applied to schizophrenia? Psychological Medicine 21, 661-673.

Shapiro, R. M. (1993). Regional neuropathology in schizophrenia: where are we? Where are we going. Schizophrenia Research 10, 187-239.

Spitzer, R. L., Endicott, J. \& Robins, E. (1978). Research Diagnostic Criteria for a Selected Group of Functional Disorders. Biometric research, New York State Psychiatric Institute: New York.

Weinberger, D. R. (1987). Implications of normal brain development for the pathogenesis of schizophrenia. Archives of General Psychiatry 44, 660-669.

Wilson, B. A., Cockburn, J. \& Baddeley, A. D. (1985). Rivermead Behavioural Memory Test. Thames Valley Test Company: Flempton, Bury St Edmunds.

Wilson, B. A., Evans, J. J., Alderman, N., Burgess, P. W. \& Emslie, H. C. (1995). Testing for executive deficits in brain injured patients. Journal of the International Neuropsychological Society 1, 164.

Wilson, B. A., Alderman, N., Burgess, P. W., Emslie, H. C. \& Evans, J. J. (1996). The Behavioural Assessment of the Dysexecutive Syndrome. Thames Valley Test Company: Flempton, Bury St Edmunds. 